\title{
AtDAT1 Is a Key Enzyme of D-Amino Acid Stimulated Ethylene Production in Arabidopsis thaliana
}

\author{
Juan Suarez ${ }^{\dagger}$, Claudia Hener ${ }^{\dagger}$, Vivien-Alisa Lehnhardt, Sabine Hummel, Mark Stahl \\ and Üner Kolukisaoglu*
}

Center for Plant Molecular Biology (ZMBP), University of Tübingen, Tübingen, Germany

\section{OPEN ACCESS}

Edited by:

Anna N. Stepanova,

North Carolina State University,

United States

Reviewed by:

Jin-Song Zhang,

Institute of Genetics and

Developmental Biology (CAS), China

Bram Van De Poel,

KU Leuven, Belgium

*Correspondence:

Üner Kolukisaoglu

uener.kolukisaoglu@zmbp.

uni-tuebingen.de

${ }^{+}$These authors share first authorship

Specialty section:

This article was submitted to

Plant Physiology,

a section of the journal

Frontiers in Plant Science

Received: 26 July 2019 Accepted: 15 November 2019

Published: 12 December 2019

Citation:

Suarez J, Hener $C$

Lehnhardt V-A, Hummel S,

Stahl M and Kolukisaoglu Ü (2019) AtDAT1 Is a Key Enzyme of D-Amino Acid Stimulated Ethylene Production in Arabidopsis thaliana.

Front. Plant Sci. 10:1609.

doi: 10.3389/fp/s.2019.01609
D-Enantiomers of proteinogenic amino acids (D-AAs) are found ubiquitously, but the knowledge about their metabolism and functions in plants is scarce. A long forgotten phenomenon in this regard is the D-AA-stimulated ethylene production in plants. As a starting point to investigate this effect, the Arabidopsis accession Landsberg erecta (Ler) got into focus as it was found defective in metabolizing D-AAs. Combining genetics and molecular biology of T-DNA insertion lines and natural variants together with biochemical and physiological approaches, we could identify AtDAT1 as a major D-AA transaminase in Arabidopsis. Atdat1 loss-of-function mutants and Arabidopsis accessions with defective AtDAT1 alleles were unable to produce the metabolites of D-Met, D-Ala, D-Glu, and L-Met. This result corroborates the biochemical characterization, which showed highest activity of AtDAT1 using D-Met as a substrate. Germination of seedlings in light and dark led to enhanced growth inhibition of atdat1 mutants on D-Met. Ethylene measurements revealed an increased D-AA stimulated ethylene production in these mutants. According to initial working models of this phenomenon, D-Met is preferentially malonylated instead of the ethylene precursor 1-aminocyclopropane-1-carboxylic acid (ACC). This decrease of ACC degradation should then lead to the increase of ethylene production. We could observe a reciprocal relation of malonylated methionine and ACC upon D-Met application and significantly more malonyl-methionine in atdat1 mutants. Unexpectedly, the malonyl-ACC levels did not differ between mutants and wild type. With AtDAT1, the first central enzyme of plant D-AA metabolism was characterized biochemically and physiologically. The specific effects of DMet on ACC metabolism, ethylene production, and plant development of dat1 mutants unraveled the impact of AtDAT1 on these processes; however, they are not in full accordance to previous working models. Instead, our results imply the influence of additional factors or processes on D-AA-stimulated ethylene production, which await to be uncovered.

Keywords: D-amino acids in plants, D-amino acid-stimulated ethylene production, D-amino acid specific transaminase, D-methionine, 1-aminocyclopropane-1-carboxylic acid, ethylene, amino acid malonylation 


\section{INTRODUCTION}

It is widely accepted that proteinogenic L-amino acids (L-AAs) are essential in all kingdoms of life, both as primary metabolites as well as elementary building blocks of proteins. In contrast, the metabolism and functions of the D-forms of amino acids (DAAs) are far less clear. Major reasons for this discrepancy are the large diversity and different functions of D-AAs in organisms. For instance, bioactive peptides like octopine from octopus and scallop, antibiotics from bacteria, and opioids from frogs were among the first substances reported to contain D-AAs (Fujii, 2002; Martínez-Rodríguez et al., 2010; Ollivaux et al., 2014). In humans, several proteins related to diseases like arteriosclerosis, Alzheimer, or Parkinson contain D-AAs, especially D-Asp that are generated by racemization of the corresponding L-AA (Fujii et al., 2011). Various free D-AAs were detected in different tissues and fluids of humans and other mammals (Hamase et al., 2002; Hamase, 2007). The most prominent example in this respect is the impact of D-Asp and D-Ser on the functions of the N-methyl-D-aspartate (NMDA) receptor in mammals: Aberrant levels of these D-AAs seem to be connected with psychological disorders and diseases of the endocrine system [for reviews, see Fuchs et al. (2005); D'aniello, 2007; Katane and Homma (2011); Balu and Coyle (2015)].

Far less is known about the metabolism and functions of DAAs in plants. This is astonishing against the background that plant roots are surrounded by D-AAs, mainly D-Ala and D-Glu, as degradation products of the peptidoglycan layer of bacterial cell walls (Dworkin, 2014). Thus, the amount of D-AAs in the rhizosphere can be more than $10 \%$ of the corresponding Lenantiomer (Brodowski et al., 2005; Amelung et al., 2006). This led to the question if D-AAs are actively utilized by plants. For a long time, D-AAs were considered as toxins due to the fact that some of them inhibit seedling growth in submillimolar concentrations (Erikson et al., 2004; Forsum et al., 2008). However, several reports suggested that D-AAs take up a similarly crucial position in plants as in microbes and animals [for further readings about D-AAs in microbes and animals, see Konno et al. (2007) and Brückner (2011)]. For instance, the DAla amount in duckweed (Landoltia punctata) was demonstrated to increase during UV light stress (Monselise et al., 2015). Furthermore, D-Ser is involved in pollen tube growth in Arabidopsis by regulating the glutamate receptor GLR1.2, which belongs to a group of plant proteins closely related to mammalian NMDA receptors (Michard et al., 2011; Forde and Roberts, 2014). In mosses (Physcomitrella patens), D-Ala and DGlu were detected in the plastidial envelope, similar to bacterial peptidoglycan (Hirano et al., 2016). This finding and others led to the conclusion that peptidoglycan, containing D-Ala and DGlu, is an integral part of the plastidial envelope not only in cryptophytes [for a review, see Chen et al. (2018)].

The number of enzymes predicted to be specific for processing D-AAs annotated in plant genomes implies much more functions for these AAs than currently known (NaranjoOrtíz et al., 2016). However, it also raises the question about their metabolism in plants, especially how the abundance of different
D-AAs is regulated. On the one hand, their content has to be maintained at required levels to ensure their activity. On the other hand, the intracellular concentrations must be limited below toxic levels. This restriction is of specific importance due to the facts that the rhizosphere is the major natural source of DAAs for plants (Vranova et al., 2012) and that D-AAs are taken up by roots in considerable amounts (Hill et al., 2011; Gördes et al., 2013). In this respect, the question arises which processes facilitate the catabolism of D-AAs in plants.

In the course of our previous studies, D-Met got into our focus because of its highest conversion rates in almost all tested accessions of Arabidopsis thaliana except in Ler (Gördes et al., 2013), although methionine represents a relatively small portion of soil amino acids (Vranova et al., 2012). But it had been detected in soil (Amelung and Zhang, 2001), and there have also been several bacterial species isolated from soil that are specialized to the utilization of D-Met as sole carbon and nitrogen source (Radkov et al., 2016). Furthermore, it is produced by different bacteria, incorporated into their cell wall and even released to their environment in order to disassemble biofilms [for a review, see Cava et al. (2011)]. Nevertheless, DMet has not been reported yet to be produced by plants.

More than 30 years ago, it was reported that feeding D-Met and other D-AAs to seedlings of cocklebur (Xanthium pennsylvanicum), pumpkin (Cucurbita moschata), sunflower (Helianthus annuus), mung bean (Vigna radiata), water melon (Citrullus vulgaris), and pea (Pisum sativum) leads to increased ethylene production (Satoh and Esashi, 1980; Liu et al., 1983; Kionka and Amrhein, 1984). This phenomenon was characterized as "D-amino-acid-stimulated ethylene production" (Satoh and Esashi, 1980). The authors tried to explain the effect by competitive malonylation of D-Met and 1aminocyclopropane-1-carboxylic acid (ACC), the precursor of ethylene. According to this hypothesis, D-Met would compete with ACC for the same malonyl transferase (Liu et al., 1983; Ling-Yuan et al., 1985; Benichou et al., 1995; Wu et al., 1995), which would lead to an increase of ACC level and subsequently ethylene production (Yang and Hoffman, 1984). However, this hypothesis could not be verified because the corresponding malonyl transferase has not been identified to date.

As shown previously, Arabidopsis plants are able to convert particular D-AAs like D-Met, D-Trp, D-Phe, and D-His to their respective L-enantiomers (Gördes et al., 2011). Additionally, the feeding of almost all tested D-AAs led mainly to the formation of $\mathrm{D}$-Ala and D-Glu. In contrast, the Arabidopsis accession Landsberg erecta (Ler) is incapable of both the D-AA to L-AA and the D-AA to D-Ala/D-Glu conversion (Gördes et al., 2013). These observations point to a central metabolic step, in which DAAs, with a high preference to D-Met, are converted to D-Ala and D-Glu by a D-AA specific transaminase (Vranova et al., 2012; Gördes et al., 2013).

Here, we describe the identification and characterization of Arabidopsis loss-of-function mutant alleles in the Columbia-0 (Col-0) accession for a previously characterized D-AA specific transaminase D-AAT (Funakoshi et al., 2008), which we named AtDAT1. This enzyme has been shown before to have a second 
enzymatic function as an aminodeoxychorismate lyase (ADCL) in the synthesis of $\mathrm{p}$-aminobenzoate, a folate precursor (Basset et al., 2004). Nevertheless, a physiological role could not be assigned to the AtDAT1 encoding gene in plants to date. Most interestingly, the homolog of AtDAT1 in Plasmodium falciparum also displays such a dual function and the ADCL activity is repressed by D-AAs (Magnani et al., 2013). Loss-of-function mutants of AtDAT1 showed almost identical defects as Ler in DAA metabolism, with D-Met as strongest effector. Indeed, we could show that the affected gene in Ler encodes for an almost non-functional AtDAT1 isoform. Biochemical analyses revealed that this enzyme prefers D-Met as amino donor and pyruvate over 2-oxoglutarate as amino acceptor, confirming the preferential production of D-Ala in Col-0. The discovery of AtDAT1 and its mutants gave us also the opportunity to verify the working model of D-AA-stimulated ethylene production in plants. We found that D-Met application causes significantly higher ethylene production and growth inhibition in atdat1 seedlings compared to wild type. According to the current working model, the increase in ethylene should be caused by a decrease in malonylation of ACC due to the increase of malonylD-Met, leading to a higher ACC oxidation. Although we found higher malonyl-methionine concentrations in atdat1 seedlings after D-Met application, the malonyl-ACC levels decreased equally in mutants and their respective wild type. This points to an additional, yet unraveled, mechanism regulating D-AAstimulated ethylene production in plants. Nevertheless, our findings indicate functions of D-Met in defined plant processes beyond unspecific growth inhibition.

\section{MATERIALS AND METHODS}

\section{Plant Material and Growth Conditions}

All Arabidopsis ecotypes as well as T-DNA insertion lines analyzed in this study were either provided by the Nottingham Arabidopsis Stock Centre (University of Nottingham, UK) or the Arabidopsis Biological Resource Center (University of Ohio, Columbus, $\mathrm{OH}$ ).

Seedlings for amino acid extraction and profiling were germinated in microtiter plates as described before (Gördes et al., 2013). For phenotypic analysis of seedlings and subsequent measurement of malonylated methionine and ACC in their extracts, plants were either germinated for 6 days in darkness or 10 days in light (all at $22{ }^{\circ} \mathrm{C}$ ). As solid growth media $1 / 2$ MS basal salts with $1 \%$ sucrose and $1 \%$ phytoagar, including conditional further additions (e.g., D-AAs, ACC) were applied. For all analyses of adult plants, these were grown in the greenhouse in soil.

\section{PCR Genotyping and RT-PCR Analysis of Arabidopsis Lines and Accessions}

Plant DNA for PCR analysis was extracted from seedlings or leaves of adult plants according to Edwards et al. (1991). To determine zygosity of T-DNA insertion lines, either a gene specific primer and a border primer or two gene specific primers flanking the insertion (for primer combinations and sequences see Table S1) were used in a PCR reaction with Taq polymerase from New England Biolabs (Frankfurt am Main, Germany) according to manufacturer's protocol. To determine the AtDAT1 sequence in different Arabidopsis ecotypes, the complete coding sequences were amplified from genomic DNA and cDNA as described above and the PCR products were sequenced directly by GATC (Konstanz, Germany). For cDNA synthesis RNA of 14 days old seedlings germinated in liquid media under long day conditions was extracted with the RNeasy Mini Kit from Qiagen (Düsseldorf, Germany) and cDNA was synthesized with RevertAid H Minus Reverse Transcriptase from Thermo Fisher Scientific (Karlsruhe, Germany), both according to manufacturers' protocols. This cDNA was used for cloning purposes (see below) and RT-PCR analysis.

\section{Cloning of AtDAT1 Variants for Recombinant Expression}

For cloning AtDAT1 from cDNA of Arabidopsis accessions Col-0 and Ler, the complete coding sequence was amplified with KOD DNA Polymerase from Merck Millipore (Schwalbach am Taunus, Germany) with the primer combination DAT1-Start/ DAT1-A1 (Table S1). PCR products were cloned into pENTR/ D-TOPO according to manufacturer's protocol (Thermo Fisher Scientific, Karlsruhe, Germany), leading to the constructs pENTR-AtDAT1 $1_{(\mathrm{Col}-0)}$ and pENTR-AtDAT1 $1_{(\mathrm{Ler})}$. To create AtDAT1 coding sequences with the single point mutations A77T and T303S, the previously described clones were cleaved with Pst $I$ and Not $I$, creating a $0.5 \mathrm{~kb}$ fragment. This was then ligated from pENTR-AtDAT1 $1_{(\mathrm{Col}-0)}$ to $\mathrm{pENTR}-\mathrm{AtDAT}_{(\mathrm{L} e r)}$ and vice versa, resulting in the constructs $\mathrm{pENTR}-\mathrm{AtDAT} 1_{(\mathrm{A} 77 \mathrm{~T})}$ and pENTR-AtDAT1 $1_{(\mathrm{T} 303 \mathrm{~S})}$. After sequence verification of the constructs, they were all used for LR reaction using the kit from Invitrogen (Karlsruhe, Germany) according to manufacturer's protocol into pGEX-2TM-GW (kindly provided by Bekir Ülker) for expression in E. coli with $\mathrm{N}$ terminal GST tag and C-terminal His tag. Additionally, the pENTR-AtDAT1 $1_{(\mathrm{Col}-0)}$ and pENTR-AtDAT1 $1_{(\mathrm{Ler})}$ were used for Gateway-based cloning into pUB-DEST-GFP for expression in plants with C-terminal GFP tag. pENTR-AtDAT1 $1_{(\mathrm{Col}-0)}$ was used for Gateway-based cloning into pUB-DEST (Grefen et al., 2010) for complementing AtDAT1 defective plants.

\section{Arabidopsis Transformation and Tobacco Leaf Infiltration}

All plant transformation vectors were transformed into Agrobacterium tumefaciens cv. pMP90-RK GV3101. Plant transformation was performed by floral dipping (Clough and Bent, 1998). For selection of transformants, seeds were either germinated on $1 / 2$ MS-Agar with $1 \%$ sucrose containing hygromycin or germinated on soil and sprayed with $2 \%$ 
BASTA from AgrEvo (Düsseldorf, Germany) depending on the used vector.

For tobacco leaf infiltration transformed Agrobacterium containing pUB10-GFP::DAT1 was mixed with a strain of transformed Agrobacterium for expression of the mCherry plastid marker (CD3-999 pt-rk; Nelson et al., 2007) and P19 A. tumefaciens cells into infiltration media [10 mM MES-KOH $(\mathrm{pH}$ 5.7), $10 \mathrm{mM}, \mathrm{MgCl}_{2}, 0.2 \mathrm{mM}$ Acetosyringone]. Using a syringe 1 $\mathrm{ml}$ of infiltration media with the mix of the three types of cells was infiltrated in the abaxial side of Nicotiana benthamiana leaves. Plants were then watered and kept on the lab bench for 2 days. Afterwards, single leaf discs were excised for confocal fluorescence microscopy.

\section{Fluorescence Microscopy}

Imaging was performed using a Leica laser scanning microscope SP8 with the corresponding software LCS or LASAF X (Leica Microsystems, Wetzlar, Germany). For excitation of GFP-fusion proteins, the Argon laser was used at $488 \mathrm{~nm}$ and the detection range was from 500 to $550 \mathrm{~nm}$. For m-RFP excitation was set to $561 \mathrm{~nm}$ and detection was from 600 to $650 \mathrm{~nm}$. All autofluorescence of chloroplasts was detected in the range from 670 to $725 \mathrm{~nm}$.

\section{Promoter::GUS Transgenic Analysis}

The promoter region from -677 to +11 of the genomic locus of AtDAT1 from Col-0 and Ler were amplified by PCR with the primer pair ProDAT1-SGW/ProDAT1-AGW (for sequences, see Table S1). The respective fragment was cloned into pENTR/DTOPO and then into pMDC163 (Curtis and Grossniklaus, 2003), to be transformed into Arabidopsis by Agrobacterium-mediated gene transfer.

Histochemical staining of GUS activity was analyzed in plants of the T2-generation that had been germinated on liquid media. For GUS staining seedlings and adult plants were washed in sodium phosphate buffer and afterwards incubated overnight at $37^{\circ} \mathrm{C}$ in this buffer containing $1 \mathrm{mM} \mathrm{X}$-Gluc (5-bromo-4-chloro3-indolyl-beta-D-glucuronic acid) and $0.5 \mathrm{mM} \mathrm{K}_{3} \mathrm{Fe}(\mathrm{CN})_{6}$. Afterwards chlorophyll was removed for documentation by several washings with hot ethanol.

\section{Recombinant Expression of AtDAT1 Variants in E. coli}

E. coli strain BL21(DE3) RIL was transformed with cDNA of AtDAT1 variants in pGEX-2TM-GW (see above) and grown in LB medium with appropriate antibiotics until they reached an $\mathrm{OD}_{600}$ of 0.5 . Then expression was induced by addition to a final concentration of $0.1 \mathrm{mM}$ isopropyl- $\beta$-D-galactoside (IPTG) and the culture was grown for $20 \mathrm{~h}$ at $18^{\circ} \mathrm{C}$. Afterwards cells were pelleted by centrifugation and washed once with TE buffer including $100 \mathrm{mM} \mathrm{NaCl}$. After further centrifugation, cells were resuspended in $20 \mathrm{mM}$ Tris, $\mathrm{pH} \mathrm{8}$, with Protease Inhibitor Cocktail from Biotool (Oberasbach, Germany). This suspension was sonicated and afterwards centrifuged with $18,000 \times \mathrm{g}$ to clear the crude extract from cell debris.

The recombinant His-tagged AtDAT1 protein variants from this crude extract were purified with Protino Ni-NTA agarose from Macherey-Nagel, (Weilmünster Germany) according to manufacturer's protocol. Therefore, the column was equilibrated and loaded with $10 \mathrm{mM}$ imidazole, washed with $20 \mathrm{mM}$ imidazole, and elution of His-tagged proteins was achieved with $250 \mathrm{mM}$ imidazole. Imidazole was removed by dialysis with Float-A-Lyzer Dialysis Device from Roth (Karlsruhe, Germany) in $10 \mathrm{mM}$ potassium phosphate, $\mathrm{pH} 8$. Protein content was determined with the Bio-Rad Protein Assay (Bio-Rad, München, Germany) according to manufacturer's protocol. Specific detection of His tagged proteins on a western blot was achieved with a monoclonal His Tag antibody conjugated to alkaline phosphatase (antikoerper-online.de, Aachen, Germany).

\section{Enzyme Assays to Determine D-AA Specific Aminotransferase Activity}

The standard reaction mixture with $2-\mathrm{OG}$ as amino group acceptor contained D-Ala (10 mM), 2-OG (50 mM), and pyridoxalphosphate (PLP; $50 \mu \mathrm{M})$ in potassium phosphate buffer (100 mM, pH 8). For assays with pyruvate as amino group acceptor, D-Ala and 2-OG were replaced by D-Met $(10 \mathrm{mM})$ and pyruvate $(50 \mathrm{mM})$, respectively. To determine substrate specificity, the tested D-AAs were all applied in $10 \mathrm{mM}$ concentration. All assay reactions in triplicates were started by addition of 3-8 $\mu \mathrm{g}$ of purified protein, incubated at $37^{\circ} \mathrm{C}$, and samples were taken at different time points up to $90 \mathrm{~min}$. Each sample was derivatized and the amino acids measured as described below.

For the determination of $\mathrm{K}_{\mathrm{M}}$ and $\mathrm{V}_{\max }$ values different D-Met concentrations $(0.1,0.5,1.0,2.0,5.0,10.0,20.0$, and $50.0 \mathrm{mM}$ DMet) have been incubated with the enzyme AtDAT1 and pyruvate as cosubstrate $(50 \mathrm{mM})$. Produced D-Alanine was analyzed after 0,5 , and $10 \mathrm{~min}$. With the means of three biological replicates for any D-Met concentration and time point, the slope of the time course was calculated and normalized to the protein amount used. To determine $\mathrm{K}_{\mathrm{M}}$ and $\mathrm{V}_{\max }$ values, a linearization according to Hofstee (1959) was used.

\section{Amino Acid Extraction and Determination From Plant Material}

Amino acid extraction and derivatization was performed as described before (Gördes et al., 2011). The incubation time of derivatization was elongated to $3 \mathrm{~h}$ and the derivatized liquid volume was adjusted with acetonitrile instead of methanol.

Almost all experiments were focused on the measurement of D/L-Alanine, D/L-Glutamate, and D/L-Methionine. To determine and quantify these amino acids in plant extracts and enzyme assays, standard materials were purchased from SigmaAldrich (Steinheim, Germany). Other chemicals were obtained 
in LC/MS grade from Roth (Karlsruhe, Germany). An AcquitySynaptG2 UPLC-MS system from Waters (Manchester, England) was used for quantification, operated in positive electrospray ionization mode. The mass spectrometer was operated at a capillary voltage of $3,000 \mathrm{~V}$ and a resolution of 20,000. Separation of the amino acids was carried out on a Waters Acquity $\mathrm{C}_{18} \mathrm{HSS}$ T3, $1.0 \times 150 \mathrm{~mm}, 1.8 \mu \mathrm{m}$ column with a flow rate of $50 \mu \mathrm{l} / \mathrm{min}$ and a $22 \mathrm{~min}$ gradient from $70 \%$ water to $99 \%$ methanol (both with $0.1 \%$ formic acid). For quantification, $3 \mu \mathrm{l}$ of sample was injected and a 5-point calibration from 0.125 to $1,250 \mu \mathrm{M}$ was used.

The quantification of malonyl-methionine $\left(\left[\mathrm{M}+\mathrm{H}^{+}\right] 218.022\right)$ and malonyl-ACC $\left(\left[\mathrm{M}+\mathrm{H}^{+}\right]\right.$188.050) was performed relatively using the same LC/MS system described above. However, the stationary phase was changed into a Waters Acquity $\mathrm{C}_{18} \mathrm{HSS}$ T3, $2.1 \times 100 \mathrm{~mm}, 1.8 \mu \mathrm{m}$ column, and a flow rate of $0.2 \mathrm{ml} / \mathrm{min}$ with a 15 min gradient from $99 \%$ water to $99 \%$ methanol (both with $0.1 \%$ formic acid) was used for separation. The malonylated compounds were identified by the exact mass of their molecular ion followed by a MS/MS fragmentation.

\section{Analysis of Ethylene}

For assaying ethylene production, Arabidopsis seedlings were grown in glass vials $(18 \mathrm{ml})$ containing $3 \mathrm{ml}$ solid medium (30 seedlings per vial) for 6 days. The vials were closed with rubber septa and opened once before measuring. After 30-90 min of further incubation, ethylene accumulating in the free air space was measured by gas chromatography using a gas chromatograph equipped with a flame-ionization detector (Felix et al., 1991).

\section{Statistical Evaluation}

Data were analyzed with IBM SPSS Statistics 24. Significance levels were analyzed using an independent two-sided Student's ttest. For further analyses between and within genotypes, we used an ANOVA followed by post hoc tests, Gabriel, or GamesHowell, depending on the equality of variances. For testing the homogeneity of variances, a Levene test was applied.

\section{RESULTS}

\section{AtDAT1 as a Candidate Gene for D-AAs Metabolism}

Initially, we observed the strong decrease of both D-AA to L-AA and especially D-AA to D-Ala/D-Glu conversion rates in Ler in comparison to other ecotypes (Gördes et al., 2013). According to the transamination hypothesis, the mutation of at least one DAA specific transaminase could be responsible for this metabolic phenotype. One candidate protein had been previously identified biochemically to be such an enzyme, named AtDAAT1 (Funakoshi et al., 2008). To investigate its role in planta we started to analyze T-DNA insertion lines of the corresponding gene (At5g57850; afterwards designated as AtDAT1) regarding their D-AA metabolism.
Homozygous plants of such insertion lines, SALK_011686 and SALK_111981 (denoted as dat1-1 and dat1-2, respectively; Figure 1A), were isolated and propagated for further analyses (see Table S1 for primer sequences). RT-PCR analysis of AtDAT1 expression displayed no transcripts with the given primer combination in dat1-1 and dat1-2 mutants compared to the corresponding wild type (Col-0) (Figure 1B). As observed previously (Lempe et al., 2005), the AtDAT1 transcript level in Ler seedlings was similar to that of wild-type Col-0. Feeding with D-Met caused the highest accumulation of D-Ala, D-Glu, and its respective L-enantiomer in Col-0 seedlings of all tested D-AAs. Therefore, seedlings of the dat1-1 and dat1-2 mutants, Col-0 and Ler were grown for 14 days on liquid $1 / 2$ MS medium in light, then supplemented with D-Met and subsequently analyzed for their AA contents. In sharp contrast to Col-0, both AtDAT1 insertion mutants were neither able to produce D-Ala, D-Glu, nor additional L-Met after application of D-Met. This AA profile was similar to that found in seedlings of the Ler accession (Figure 1C).

Further in silico analyses of public transcriptomic data (Lempe et al., 2005) revealed that the accession M7323S displayed a strongly reduced AtDAT1 transcript level, which could be confirmed by RT-PCR (Figure 1B). When this accession was grown on D-Met supplemented medium, defects in AA metabolism were observed (Figure S1) similar to those found in Ler and the dat1 mutant seedlings. This defect was not just due to the reduced transcription of AtDAT1 in M7323S. Sequencing of the genomic locus and the cDNA of AtDAT1 from M7323S revealed that this gene contains a $\mathrm{T} \rightarrow \mathrm{A}$ mutation at genomic position +1259 . This leads to a nonsense mutation at the third position of a cysteine codon (TGT) to a stop codon (TGA) at position 248 of the AA sequence (C248STOP) (Figure 1A). In contrast, sequencing of the genomic locus and the cDNA of AtDAT1 from Ler revealed two missense mutations leading to AA exchanges of the protein sequence (A77T and T303S) (Figure 1A).

To examine whether these mutations in the AtDAT1 Ler allele are responsible for the metabolic aberrations in this accession, we performed different genetic approaches. First, ubiquitin promoter-driven expression of the AtDAT1 Col-0 allele in transgenic Ler plants led to the reconstitution of the D-Met metabolism in Ler and its complementation in the dat1-2 mutant (Figure 2A). Second, F1 seedlings derived from crosses between Col-0 and Ler and between Col-0 and dat1-2 displayed no defects in D-Met metabolism as observed in Ler and dat1-2, irrespective of the maternal origin, whereas the offspring of the Ler $\mathrm{x}$ dat1-2 crossing did (Figure 2B). These data prove the defect of AtDAT1 function in the Ler accession and the dat1-2 insertion mutant.

To answer the remaining question about the reason for this defect in Ler, the expression of AtDAT1 was analyzed. As mentioned before, the AtDAT1 transcript levels appeared similar in Col-0 and Ler (Figure 1B). This observation was supported by analysis of transgenic plants containing the uidA reporter gene (GUS) under the control of the AtDAT1 promoter either from the Col-0 or Ler allele (Figures S2A, B). There, it can be seen that the reporter constructs are active in seedlings and 

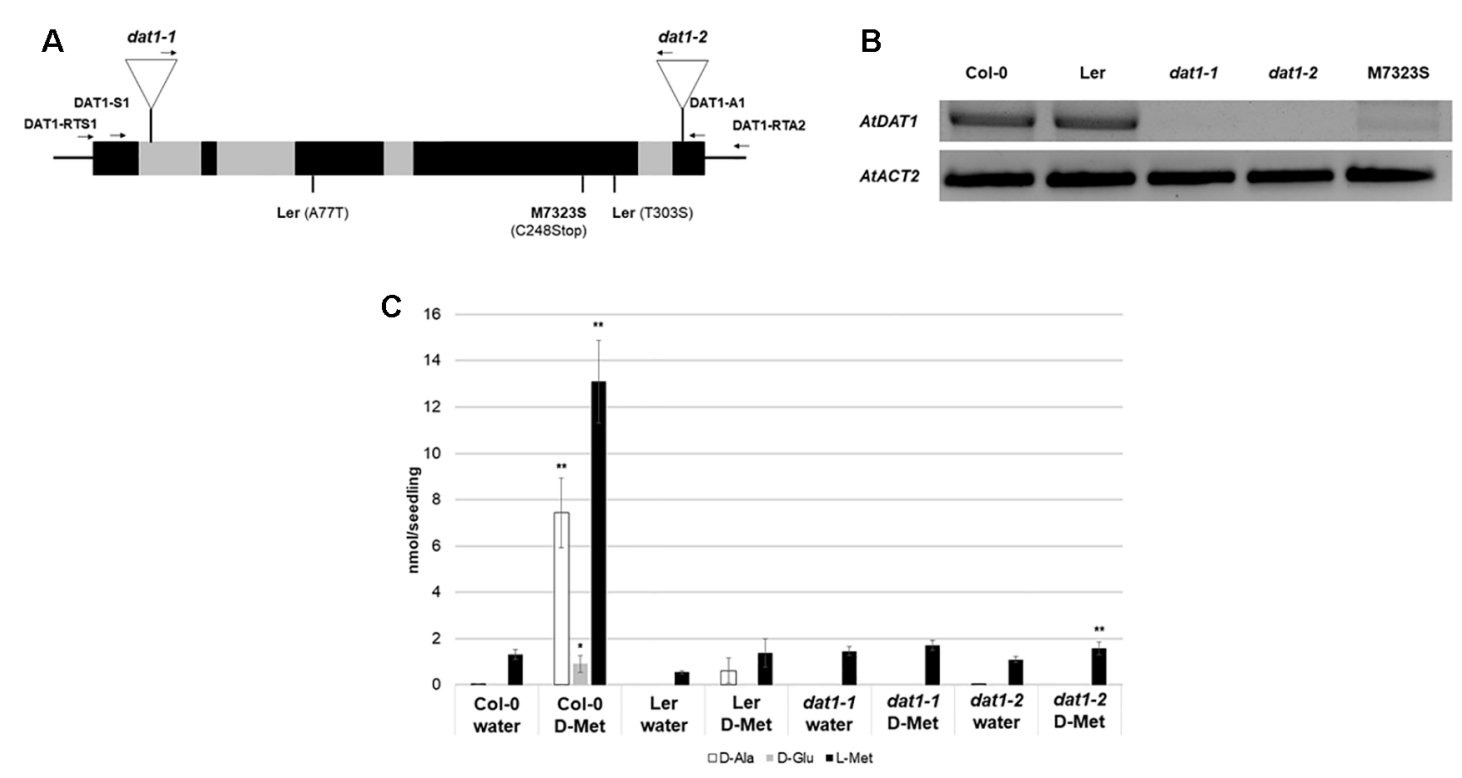

FIGURE 1 | AtDAT1 as a candidate protein for the metabolism of D-AAs in Arabidopsis. (A) Scheme of the genomic structure of AtDAT1 (exons and introns in black and grey, respectively) with the positions of T-DNA insertions in dat1-1 and dat1-2 as well as the mutations found in Ler and M7323S. Arrows indicate primers used for genotyping the T-DNA insertions and RT-PCR (for primer sequences, see Table S1). (B) RT-PCR analysis of AtDAT1 expression in Col-0, Ler, dat1-1, dat1-2, and M7323S (top: AtDAT1; bottom: AtACT2). (C) Contents of D-Ala (white), D-Glu (gray), and L-Met (black) in seedlings of Col-0, Ler, dat1-1, and dat1-2 without (water) and with D-Met treatment for $16 \mathrm{~h}$ (D-Met). For each measurement four seedlings were pooled and further processed. Error bars represent the standard deviation from three independent measurements. The asterisks indicate the significance level (t-test) of differences of all measurements to the respective line without D-Met treatment $\left({ }^{*} \mathrm{p}<0.05 ;{ }^{* *} \mathrm{p}<0.01\right)$.
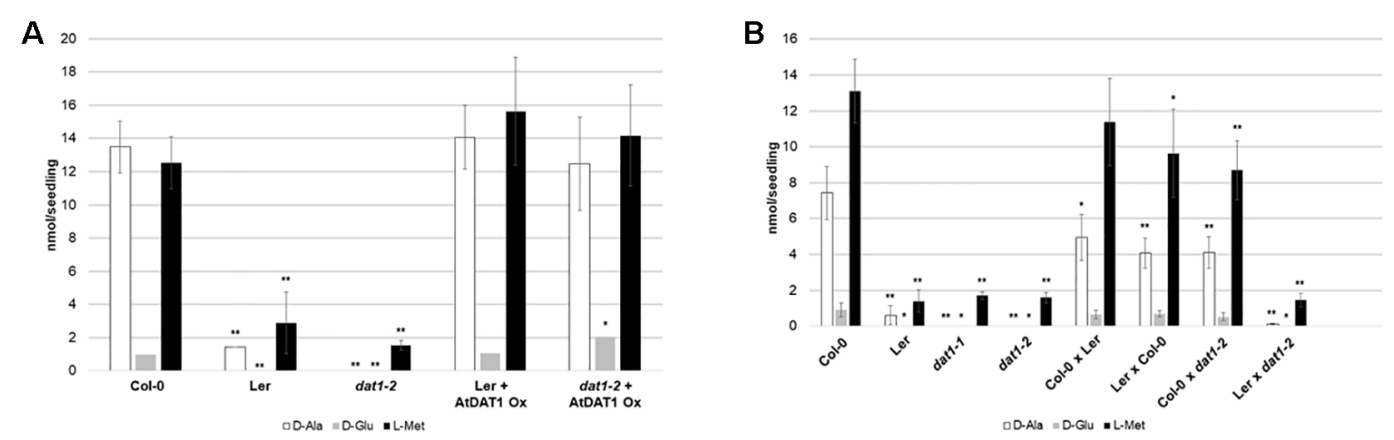

FIGURE 2 | D-Met metabolism in lines overexpressing AtDAT1 and in F1 seedlings from crosses of Col-0, Ler and dat1-2. Contents of D-Ala, D-Glu, and L-Met after overnight exposure to D-Met (A) in Ler and dat1-2 seedlings overexpressing AtDAT1 (AtDAT1 Ox) and their corresponding background lines and (B) in seedlings of $\mathrm{F} 1$ progeny of crosses of Col- 0 , Ler, and dat1-2 and their corresponding parental lines; for further information, see Figure 1 C $\left({ }^{*} \mathrm{P}<0.05\right.$; $\left.{ }^{* *} \mathrm{p}<0.01\right)$.

adult plants and with less GUS staining in late floral stages and seeds (Figure S2A), corresponding to expression patterns displayed in the eFP browser (Winter et al., 2007). The activity of the AtDAT1 promoters derived from Col- 0 and Ler showed no apparent differences, irrespective of the presence of L-Met or DMet in the media (Figure S2B). Subcellular mislocalization would have been another explanation for affected AtDAT1 function in Ler. Therefore, GFP-tagged AtDAT1 gene variants derived from cDNA of both ecotypes expressed under the control of the ubiquitin 10 promoter were transiently transformed into tobacco leaves (Figure S3). The Col-0 as well as the Ler cDNA derived AtDAT1 fusion proteins localized to the chloroplasts, as it had been shown before for GFP-tagged AtDAT1 $1_{(\mathrm{Col}-0)}$ (Basset et al., 2004). Therefore, a possible misexpression of AtDAT1 or its mis-localization of AtDAT1-GFP in Ler does not cause the aberrant D-Met metabolism in this accession.

\section{A Missense Mutation of the AtDAT1 Ler Allele Leads to an Almost Complete Loss of the Enzymatic Activity}

To clarify if the enzyme encoded by the Ler AtDAT1 allele is able to transaminate D-AAs, the Ler $\left(\operatorname{AtDAT}_{(\mathrm{L} e r)}\right)$ and Col-0 $\left(\operatorname{AtDAT1}_{(\mathrm{Col}-0)}\right)$ versions of AtDAT1 were expressed with an 
N-terminal GST-tag in E. coli. After purification by affinity chromatography (for purification results, see Figure S4), their enzymatic activities were tested according to Funakoshi et al. (2008).

We first tested AtDAT1 (Col-0) for its capability to transaminate 2-oxoglutarate (2-OG) or pyruvate using 16 different D-AAs as amino group donors. With 2-OG used as amino group acceptor, a transaminase reaction was only detectable for the donors D-Met, D-Trp, and D-Ala (Table S2), whereas with pyruvate as acceptor, almost all D-AAs, with the exception of D-Pro, led to the formation of D-Ala (Figure 3). Furthermore, we measured an over 100 times higher activity for the enzymatic reaction with pyruvate as acceptor than with 2OG, irrespective of the D-AA applied as amino group donor (Table S2). The comparison of the AtDAT1 ${ }_{(\mathrm{Col}-0)}$ activities using different D-AAs and pyruvate as substrates revealed that D-Met was the best tested amino group donor (Figure 3). Using pyruvate and D-Met as substrates, we determined the $\mathrm{K}_{\mathrm{M}}$ and $\mathrm{V}_{\max }$ of AtDAT1 $1_{(\mathrm{Col}-0)}$ to be $17.4 \mathrm{mM}$ and 0.07 nkat, respectively.

To characterize the activity of $\operatorname{AtDAT}_{(\mathrm{Ler})}$ in comparison to AtDAT1 $1_{(\mathrm{Col}-0)}$, enzymatic assays were performed with two substrate combinations: first, with D-Met as amino group donor and pyruvate as acceptor, respectively, as the best substrate combination for AtDAT1 $1_{(\mathrm{Col}-0)}$ and, second, with DAla as amino group donor and 2-OG as acceptor. As shown in Figures $4 \mathrm{~A}$ and $\mathrm{B}$ for both substrate combinations, the activity of $\operatorname{AtDAT} 1_{(\mathrm{Ler})}$ dropped to $0-5 \%$ compared to that of $\operatorname{AtDAT} 1_{(\mathrm{Col}-0)}$.

We next addressed the question whether only one of the missense mutations in AtDAT1 $1_{(\text {Ler })}$ (A77T or T303S) is sufficient to cause the activity loss. The alignment of DAT1 amino acid sequences from different plant species revealed that the alanine at position 77 is more conserved than the threonine at position 303 (Figure S5). To analyze the impact of the mutations, AtDAT1 (Col-0) derived isoforms harboring single amino acid exchanges of AtDAT1 $1_{(\text {Ler })}$ were also expressed as N-terminal GST fusions in $E$. coli. The recombinant proteins were affinity-purified and tested for their activity. The enzyme isoform with the T303S amino acid exchange AtDAT1 $1_{(\mathrm{T} 303 \mathrm{~S})}$ showed an activity comparable to $\operatorname{AtDAT1}_{(\mathrm{Col}-0)}$ (Figures 4A, B). In contrast, the mutation A77T led to a strong decrease in the production of D-Glu (Figure 4A) and D-Ala (Figure 4B) with 2-oxoglutarate or pyruvate as substrates, respectively. Instead, the enzymatic defect of AtDAT1 $1_{(\mathrm{A} 77 \mathrm{~T})}$ was quantitatively similar to that of AtDAT1 (Ler). From these data, we conclude that solely the A77T amino acid exchange is responsible for the activity loss of $\operatorname{AtDAT} 1_{(\text {Ler })}$. Furthermore, the enzymatic data also revealed that the Ler variant of AtDAT1 is not completely inactive with about $5 \%$ remaining activity in comparison to Col-0 (Figure 4B).

\section{The Loss of AtDAT1 Leads to Decreased Seedling Growth in Response to D-Met}

After identification of AtDAT1 as a central enzyme of D-AA metabolism, the question arose whether the loss of AtDAT1 gene function leads to defects in Arabidopsis growth and development. Under greenhouse conditions in soil growth of dat1-1 and dat1-2 mutant plants could not be distinguished from Col-0 (Figure S6). We next asked of how the mutant lines and Ler would grow in presence of D-Met. Growth of dat1-1 and dat1-2 seedlings on

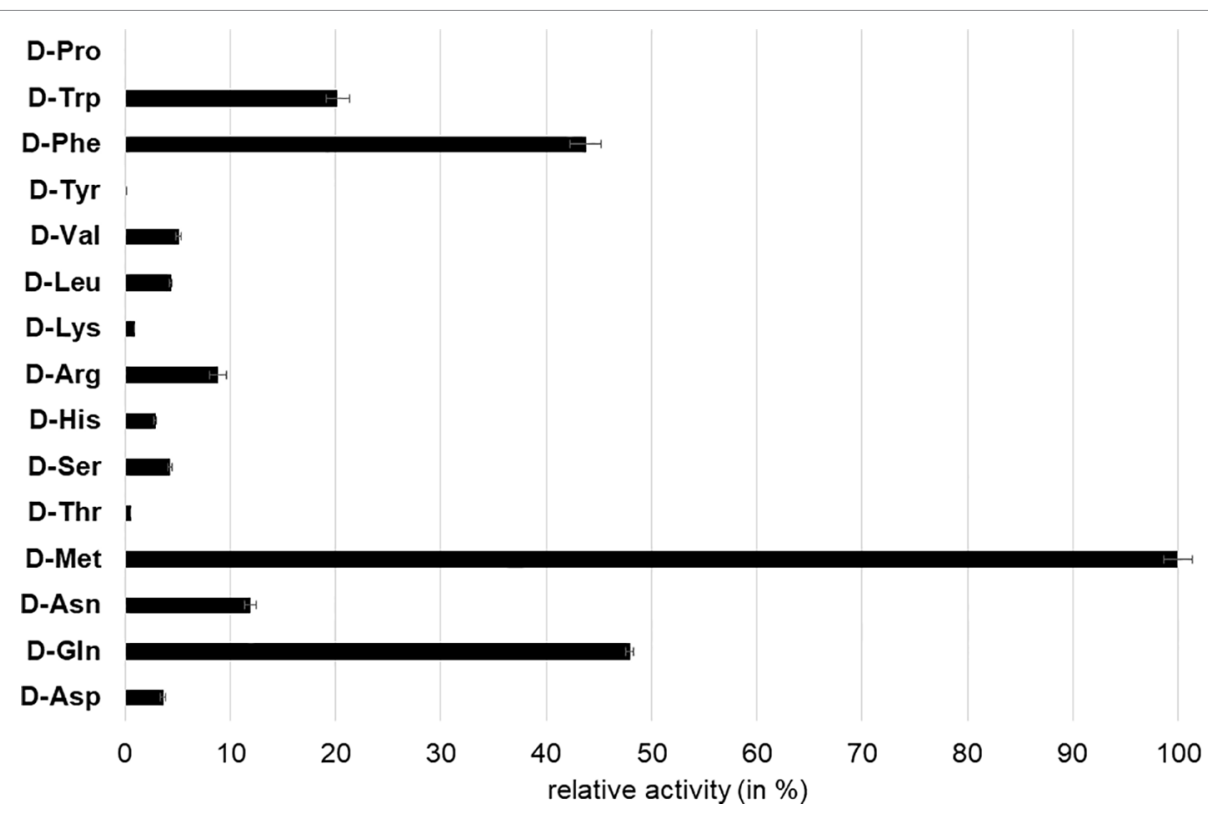

FIGURE 3 | Relative D-Ala producing activity of AtDAT1 with different D-AAs as amino group donor and pyruvate as acceptor. Activity of reaction with D-Met was set to $100 \%$ and all other reactions were calculated in relation to it. Each bar represents the mean of measurement of three independent assays. Error bars $( \pm$ SD). 

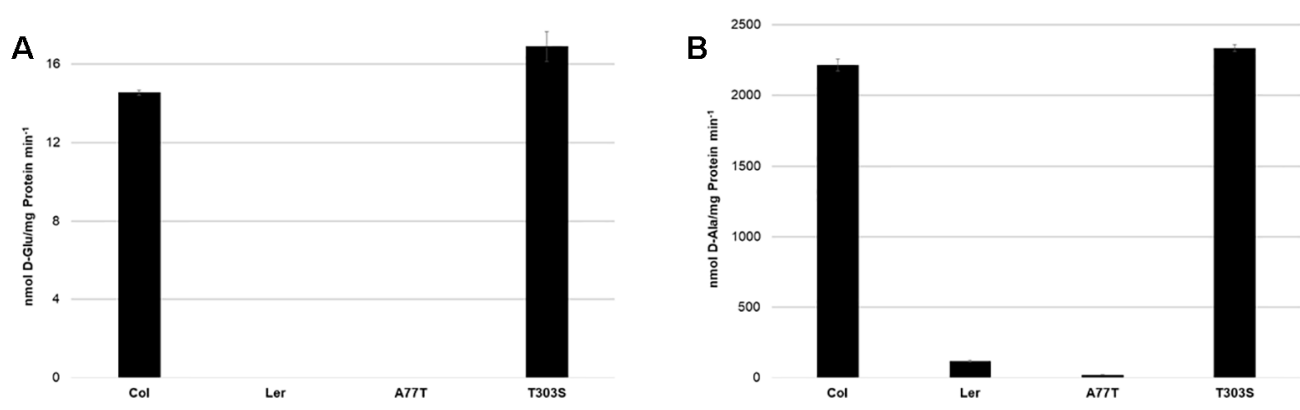

FIGURE 4 | Activities of AtDAT1 variants. Transaminase activities of AtDAT1 (Col-0) $_{\text {, AtDAT1 }}$ (Ler), AtDAT1 (A77T), and AtDAT1 $_{(T 303 S)}$ with D-Met as amino group donor and (A) 2-oxoglutarate or (B) pyruvate as acceptor molecule are displayed; for further information, see Figure 3.

media containing $500 \mu \mathrm{M}$ D-Met resulted in a retardation compared to the corresponding wild type, whereas Ler took an intermediate response (Figure 5A). Testing this growth behavior on the dark-grown etiolated seedlings revealed an even more pronounced growth difference between the dat 1 mutants and Col-0 (Figure 5B). All these growth differences were specific for D-Met, whereas the addition of the same concentrations of LMet did not lead to these differential effects (Figure 5A). Altogether, D-Met inhibited seedling growth specifically in AtDAT1 affected lines.

The reduced growth of hypocotyls and roots of dat1-1 and dat1-2 seedlings, especially in the dark (Figure 5A), reminded of phenotypes caused by the gaseous plant hormone ethylene. This gets even clearer with a look on the hypocotyl length of the four dark grown lines (Figure 5B): There was a highly significant decrease of dat1-1 and dat1-2 hypocotyl length of about oneeighth compared to Col-0 grown on $500 \mu \mathrm{M}$ D-Met. Although increasing L-Met concentrations also led to shorter hypocotyls, this effect was similar in mutant and wild-type plants. Furthermore, the growth inhibition was by far not as strong as with D-Met (Figure S7).

\section{AtDAT1 Mutants Display Enhanced D-AA Stimulated Ethylene Production}

To test whether ethylene synthesis is indeed affected in dat 1 mutants by D-Met, we added $\alpha$-aminoisobutyric acid (AIB) to the growth medium, which leads to the inhibition of ACC oxidase, the enzyme catalyzing the last step of ethylene synthesis (Satoh and Esashi, 1980). As shown in Figure 6A, the addition of $2 \mathrm{mM}$ AIB to the growth medium led to a reversion of growth reduction by D-Met of all dat1 affected lines in the dark. This indicates that the increased ethylene production in these lines is caused by D-Met in the medium.

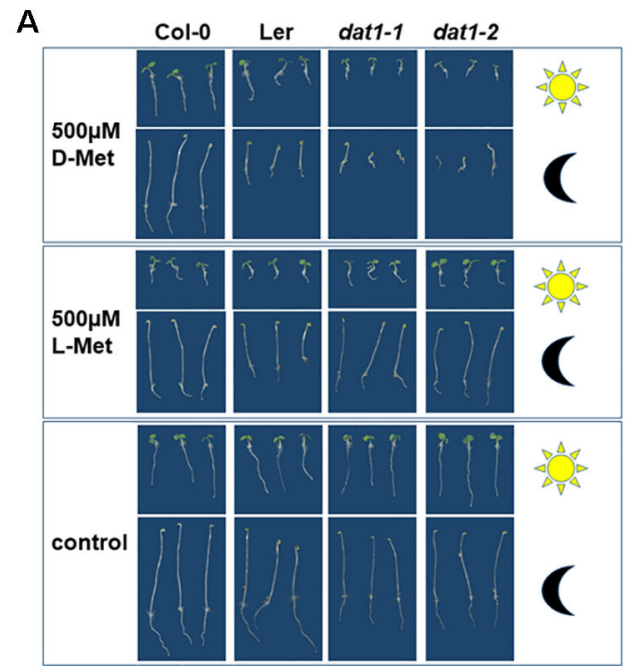

B

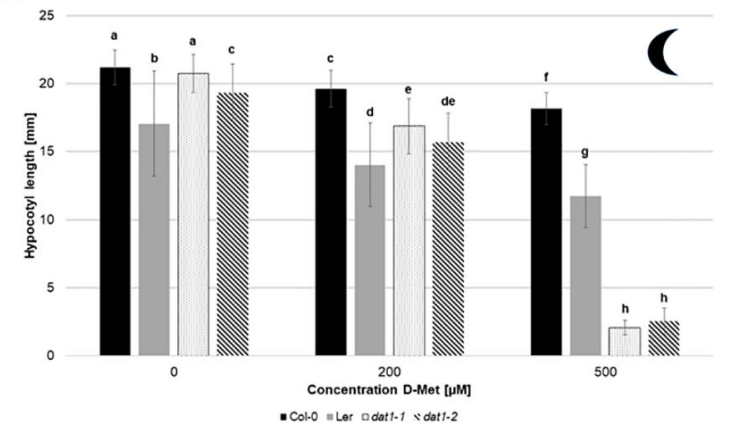

FIGURE 5 | Seedling growth is differentially suppressed by D-Met in AtDAT1 knock out-lines. (A) Seeds of Col-0, dat1-1, and dat1-2, and Ler were germinated either in continuous light (sun) or darkness (moon) on different solid growth media (with $500 \mu \mathrm{M}$ D-Met, with $500 \mu \mathrm{M} \mathrm{L-Met} \mathrm{supplemented} \mathrm{or} \mathrm{without}$ supplementation). (B) Hypocotyl growth of the before mentioned dark grown plants. The bars (Col-0: black, Ler: grey, dat1-1: dotted, dat1-2: striped; $\mathrm{n}=30$ ) represent the average hypocotyl length. Different letters indicate statistically significant differences $(p<0.05)$ tested by an ANOVA. Error bars $( \pm$ SD). 


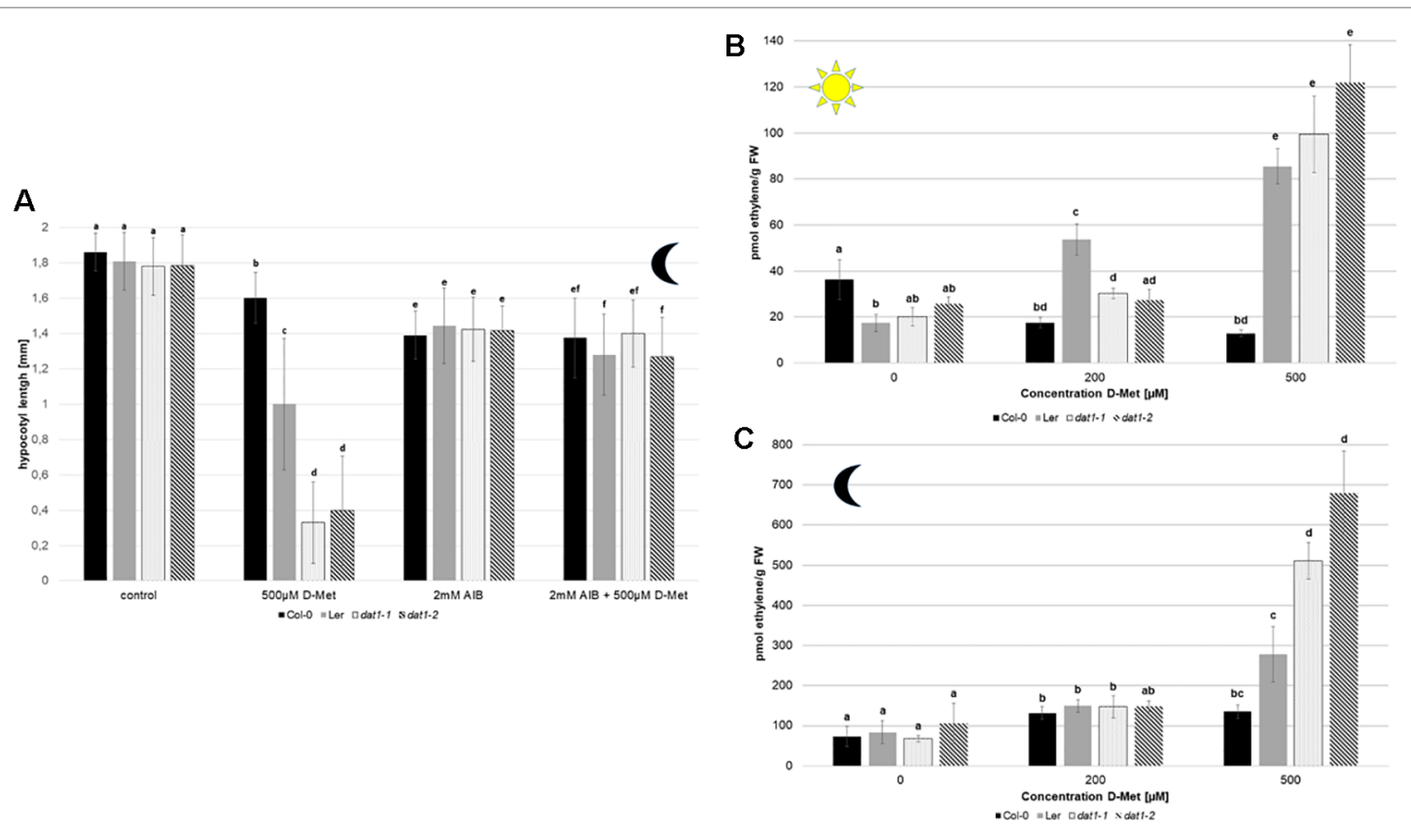

FIGURE 6 | D-Met leads to an increase of ethylene in AtDAT1 knock out-lines. (A) Seeds of Col-0, dat1-1, and dat1-2, and Ler were germinated in continuous darkness on solid growth media without any supplementation (control), supplemented just with 500 HM D-Met, supplemented just with 2 mM AIB, and supplemented with both agents together. For further information, see Figure 5B. (B) Ethylene contents in seedlings of Col-0, Ler, dat1-1, and dat1-2 were measured after growth in continuous light or (C) in darkness in vials with solid growth media supplemented with 200 and 500 4 M D-Met, and additionally without supplementation. The bars (Col-0: black, Ler: gray, dat1-1: dotted, dat1-2: striped) represent the average values of three biological replicates. Different letters indicate statistically significant differences $(p<0.05)$ tested by an ANOVA. Error bars $( \pm S D)$.

To elucidate if ethylene production was indeed altered, we measured its content in Ler, the dat 1 mutants, and Col- 0 grown in continuous light and dark. The addition of $500 \mu \mathrm{M}$ D-Met was sufficient to induce a significant increase of up to threefold of ethylene production in light grown Ler and dat1 mutants compared to Col-0 (Figure 6B). Even stronger changes in ethylene production could be observed for both dat 1 mutant seedlings grown in the presence of D-Met in the dark, whereas Ler displayed again an intermediate phenotype (Figure 6C).

As mentioned above, the increase of ethylene production by $\mathrm{D}$-AAs was attributed to competitive malonylation of D-AAs instead of ACC, which should lead to enhanced ACC oxidation resulting in higher ethylene concentration (Yang and Hoffman, 1984). To verify this assumption, we measured the contents of malonyl-methionine and malonyl-ACC in D-Met treated seedlings. In these measurements, we detected a significant increase of malonyl-methionine in Col-0, Ler, and dat1 seedlings upon D-Met treatment (Figures 7A, B). This accumulation was far higher (up to fivefold) in the dat1 mutants compared to the corresponding wild type, irrespective of the light regime (Figures 7A, B). Furthermore, Ler also showed a D-Met induced over-accumulation of malonylmethionine in the light (Figure $\mathbf{7 A}$ ), but not in darkness (Figure 7B).

Since the amount of malonyl-ACC in these experiments was below our detection limit, we added $10 \mu \mathrm{M}$ ACC to the media and measured the malonyl-ACC in the seedlings. In this case, we were able to detect large amounts of malonyl-ACC in the seedlings of all genotypes, which decreased drastically upon DMet addition (Figures 8A, B). It must be noted that the treatment of seedlings with 200 or $500 \mu \mathrm{M}$ D-Met together with $10 \mu \mathrm{M}$ ACC is relatively extreme and probably does not reflect physiological conditions. However, D-Met induced malonyl-ACC reduction was undue to production of malonylmethionine caused by ACC, which was comparable with and without ACC addition (Table S3). Nevertheless, there was no significant difference of malonyl-ACC reduction of Ler and dat1 mutants to Col-0 at higher D-Met concentrations (Figures 8A, B).

\section{DISCUSSION}

For several decades, the detrimental, but partially also beneficial, effects of D-AAs on plants have been investigated (Valdovinos and Muir, 1965; Aldag and Young, 1970; Erikson et al., 2004; Erikson et al., 2005; Gördes et al., 2011; Hill et al., 2011). It is noteworthy, that there are reports of some D-AAs synthesized de novo by plants (Brückner and Westhauser, 2003; Strauch et al., 2015). However, there is growing evidence in recent years that almost all D-enantiomers of proteinogenic L-AAs are taken up by plants (Aldag and Young, 1970; Forsum et al., 2008; Gördes et al., 2011; Hill et al., 2011) and also metabolized to significant 

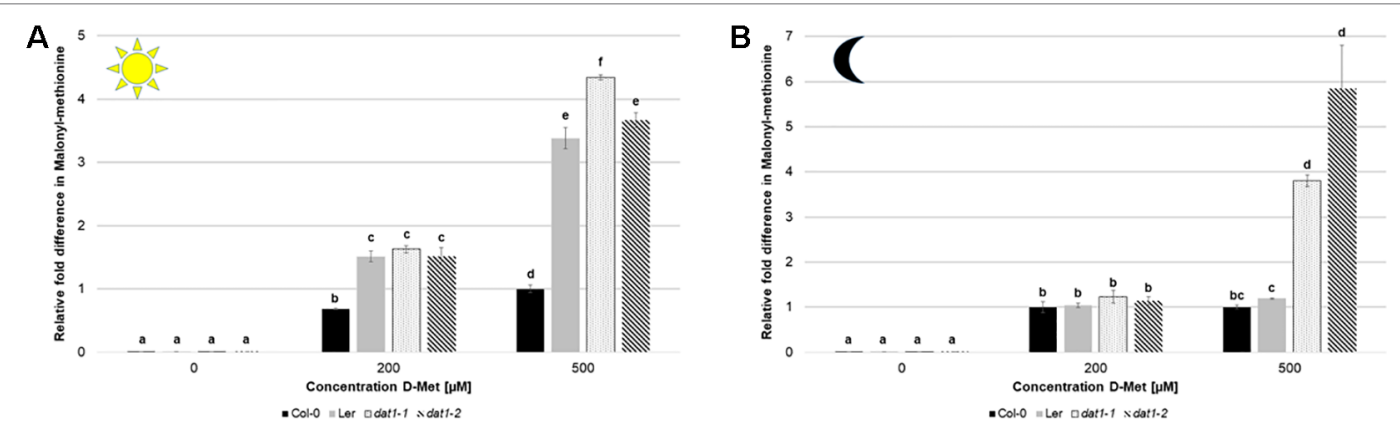

FIGURE 7 | D-Met affects formation of malonyl-methionine differently in Col-0, Ler, and dat1 mutants. Malonyl-methionine contents in seedlings of Col-0, Ler, dat11, and dat1-2 were measured after growth (A) in continuous light or (B) in darkness on agar plates supplemented with 200 and $500 \mu$ M D-Met, and additionally without supplementation. The relative values are given in fold changes with the values of Col-0 at $500 \mu \mathrm{M}$ D-Met set to 1. The bars (Col-0: black, Ler: gray, dat1-1: dotted, dat1-2: striped) represent the average values of three biological replicates. Different letters indicate statistically significant differences $(p<0.05)$ tested by an ANOVA. Error bars ( \pm SD).
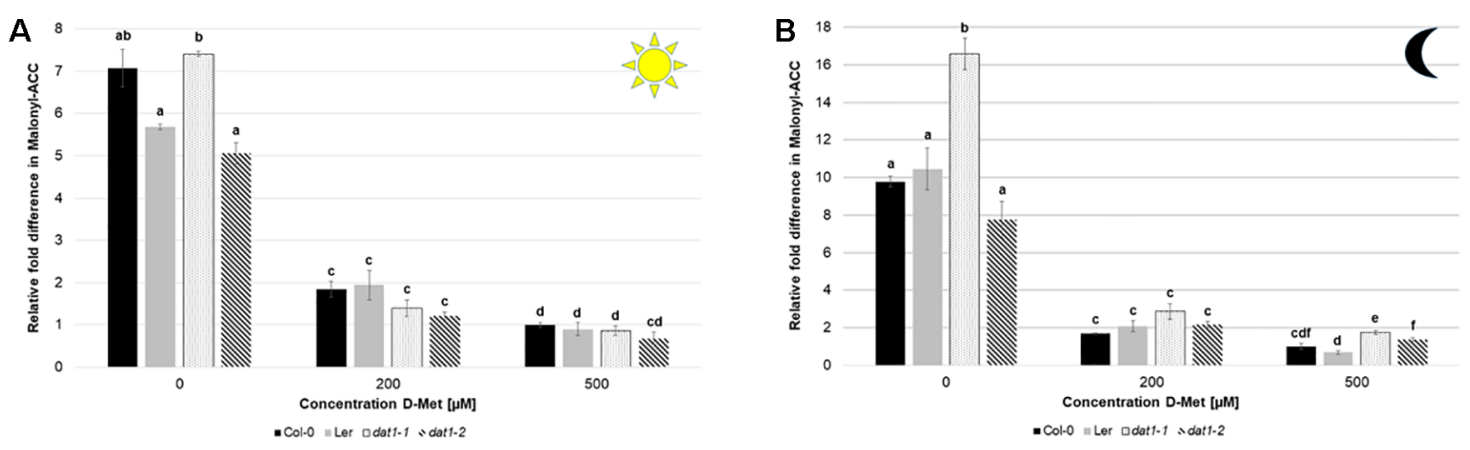

FIGURE 8 | D-Met leads to a decrease of malonyl-ACC levels in all tested lines. Malonyl-ACC contents in seedlings of Col-0, Ler, dat1-1, and dat1-2 were measured after growth (A) in continuous light or (B) in darkness on agar plates supplemented with 200 and $500 \mu M$ D-Met, and without supplementation. Additionally, all plates contained $10 \mu \mathrm{M}$ ACC. The relative values are given in fold changes with the values of Col-0 at $500 \mu \mathrm{M}$ D-Met + $10 \mu \mathrm{M}$ ACC set to 1. The bars (Col-0: black, Ler: gray, dat1-1: dotted, dat1-2: striped) represent the average values of three biological replicates. Different letters indicate statistically significant differences $(p<0.05)$ tested by an ANOVA. Error bars $( \pm S D)$.

amounts (Aldag and Young, 1970; Gördes et al., 2011). With the proof provided in the actual report, the long standing question was addressed how D-AAs are utilized in plants.

In the light of the observations of Gördes et al. (2011), three possible mechanisms for the metabolism of D-AAs in plants had been suggested: racemization, deamination, and transamination of D-AAs (Vranova et al., 2012; Gördes et al., 2013). Our data indicate that transamination by AtDAT1 is responsible for major steps of D-AA turnover in Arabidopsis. This is reflected by its broad range of D-AAs transaminated, although its turnover rate and its affinity is low. Furthermore, we showed that the major product of this enzymatic reaction is $\mathrm{D}$-Ala with $\mathrm{D}$-Met as the favored amino group donor. D-Ala was also preferentially produced when plants were fed with other D-AAs. The preferred synthesis of D-Ala is caused by the preference of AtDAT1 on pyruvate over 2-OG as substrate. In comparison to the work of Funakoshi et al. (2008), who used 2-OG as amino group acceptor for their characterization of AtDAT1, our results revealed a higher $\mathrm{V}_{\max }$ with pyruvate as substrate as compared to
2-OG. Most interestingly, the different enzymatic activities with pyruvate and 2-OG as amino group acceptors with ratios of 100:1 and more were in a comparable range as the $\mathrm{D}-\mathrm{Ala} / \mathrm{D}$-Glu ratios found in plants after D-AA application (Gördes et al., 2011). Nevertheless, we could approximately substantiate the results from Funakoshi et al. (2008) when we used 2-OG as substrate in our enzymatic assays.

A major question in our studies addressed the role of AtDAT1 in D-AA stimulated ethylene production. As it is demonstrated here, this phenomenon is tightly connected to AtDAT1 (Figure 9). The loss of DAT1 leads to a significant increase of ethylene after D-Met application, resulting primarily in shortening of the hypocotyl and root in the dat1 mutants and Ler irrespective of the light regime. D-Met application also led to an increased production of malonyl-methionine, especially in the dat1 mutants and Ler, and the amount of malonyl-ACC developed reciprocally in all tested lines. The reciprocal accumulation of malonylated D-Met and ACC implies that the loss of AtDAT function or enzymatic activity results in over- 


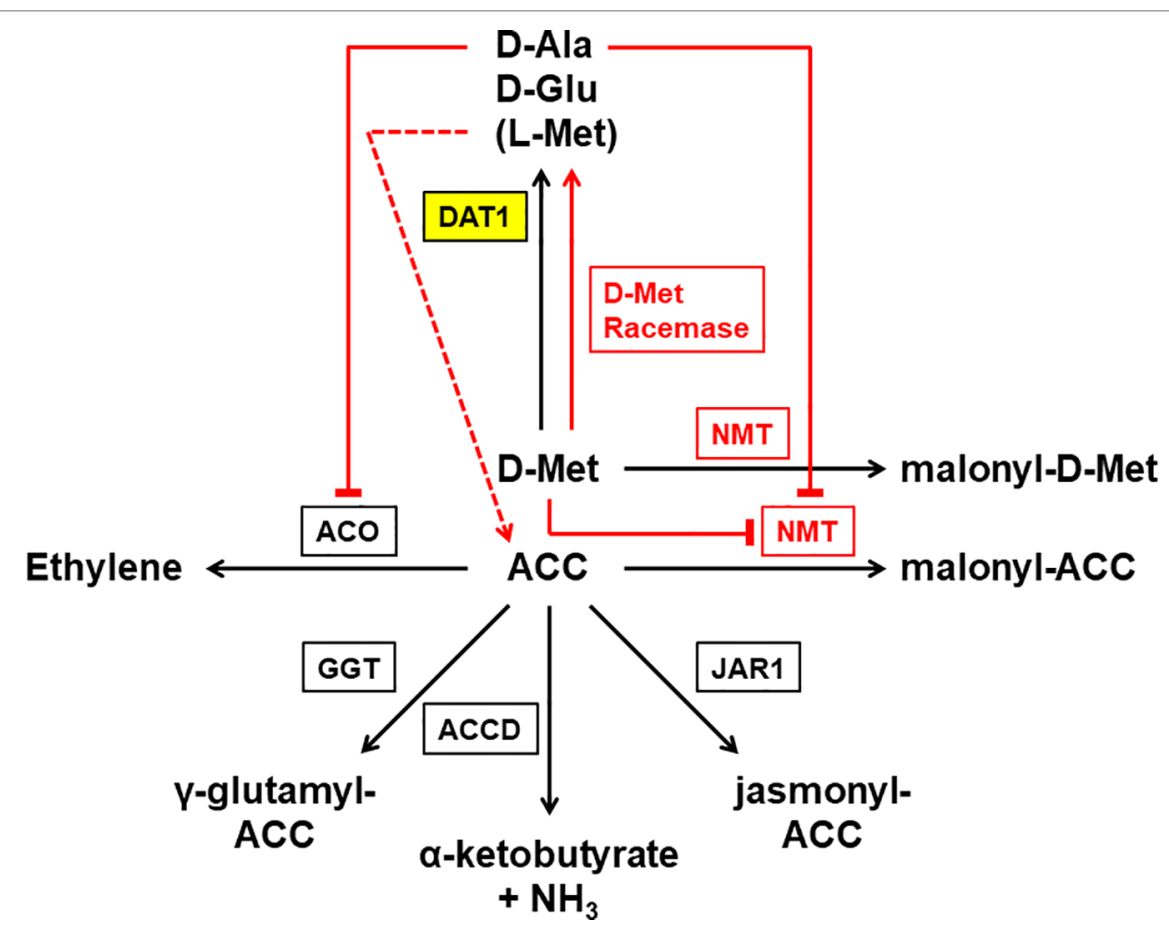

FIGURE 9 | Working model of the different reactions leading to D-Met stimulated ethylene production in plants. This reaction scheme summarizes proven (black) and postulated (red) enzymes and reactions involved in the process of D-Met stimulated ethylene production in plants. As the central enzyme of this study DAT1 is highlighted in yellow. Externally applied D-Met is mainly transaminated by a D-amino acid transaminase (DAT1) to produce D-Ala and D-Glu. Additionally, L-Met is produced that mainly results from a second transamination step after transamination of D-Met. ACC is the precursor of the gaseous hormone ethylene and this reaction is catalyzed by the ACC oxidase (ACO). Alternative to transamination, D-Met is malonylated by a N-malonyl transferase (NMT), which also uses ACC as a substrate. The malonylation of D-Met by NMT leads to the competitive repression of the reaction with ACC. The consequence of DAT1 loss of activity would be an increase of D-Met concentration, which would repress ACC malonylation and lead to increased ethylene production. Although malonylation is thought to be the major route to regulate cellular ACC concentration, there are three additional ways known: the glutamylation of ACC by the $\gamma$-glutamyl transpeptidase (GGT), the addition of jasmonic acid to ACC by jasmonic acid resistance 1 (JAR1), and the deamination of ACC by the ACC deaminase (ACCD). But also two other metabolites of D-Met may affect ACC and ethylene levels: D-Ala is able to inhibit ACO but would be missing in case of DAT1 loss. In contrast, L-Met is a precursor of ACC and may also be produced by direct racemization from D-Met. Higher concentrations of L-Met by such a racemization may also lead to an increase of ACC levels even when DAT1 activity is decreased.

accumulation of ACC that is then causing an increased ethylene production and eventually reduced seedling growth. However, this conclusion has to be reviewed critically, because even the dat1 mutant seedlings did not show the full spectrum of the canonical triple response, as tightening of the apical hook or thickening of the hypocotyl was only partially observed. Furthermore, no differences in levels of malonylated ACC were detected between D-Met treated Col-0 and dat1 mutants. However, it must be noted that in all previous studies the production of ethylene in response to malonylation of ACC and D-AAs were measured during overnight feeding experiments. The physiological growth responses and the contents of D-Met, ACC, and their malonylated derivatives in the plants over a longer period of the treatment were shown here for the first time.

The increased production of malonyl-methionine and ethylene without a decreased malonyl-ACC production in the dat 1 mutants in comparison to the control raises the question whether the original working model of D-Met stimulated ethylene production needs additional factors or metabolic processes. One explanation may be that malonylation is not the only way to regulate the ACC level in plants (Figure 9). There is also the possibility that ACC is conjugated with glutathione to $\gamma$-glutamyl-ACC (GACC) and with jasmonic acid to JA-ACC to control the ACC homeostasis. Additionally, plants can irreversibly degrade ACC to $\alpha$-ketobutyrate by an ACC deaminase [for reviews about ACC content regulation, see Van de Poel and Van Der Straeten (2014); Le Deunff and Lecourt (2016), and Vanderstraeten and Van Der Straeten (2017)]. To date, neither the contribution of each of these ACC catabolic pathways nor their interplay for the control of ACC homeostasis have been studied, yet. It remains to be investigated whether DMet, its malonylated form, or the loss of this way to degrade DMet have an impact also on the alternative ACC degradation pathways.

Another explanation would be given by a racemization of $\mathrm{D}$ Met, which has been proposed before but never been proven (Vranova et al., 2012; Gördes et al., 2013). Our results imply that the majority of the increase of L-Met in D-Met fed Col-0 plants arises from the reamination of 4-methylthio-2-oxobutanoate 
after removal of the amino group from D-Met by AtDAT1. However, direct racemization of D-Met cannot be excluded. If such a direct racemization really exists, additional L-Met would be produced irrespective of AtDAT1 activity and would be partially converted to ACC in the Yang cycle (Figure 9). This additional ACC would contribute to the increased ethylene contents in D-Met treated dat1 mutants, because ACC malonylation is inhibited competitively by higher D-Met levels.

A third explanation may be given by the effect of the AtDAT1 enzymatic products on the activity of other enzymes. Here we demonstrated that the loss of this enzyme leaves dat1 mutants without the ability to produce D-Ala, D-Glu and additional LMet in response to D-Met. Most interestingly, it was shown previously that D-Ala inhibits the ACC oxidase (ACO) (Gibson et al., 1998; Brunhuber et al., 2000; Charng et al., 2001; Thrower et al., 2006). This means that plants with functional DAT1 would malonylate D-Met instead of ACC but the produced D-Ala would partially inhibit the ACO and as one consequence the additional ACC would just be partially converted to ethylene. In the dat 1 mutants this inhibiting effect of $\mathrm{D}$-Ala would be lost and may explain the ethylene increase in these lines in comparison to the corresponding wild type. The same lack of D-Ala accumulation may also contribute to the higher content of malonyl-methionine in the dat 1 mutants and to the comparable amount of malonylated ACC in all tested lines: DAla also partially inhibits the putative malonyl transferase (Kionka and Amrhein, 1984; Liu et al., 1985; Chick and Leung, 1997). The malonylation of D-Met would then be limited by DAla in Col-0 but not in the dat1 mutant lines and Ler. If D-Met is the preferred substrate of the malonyl transferase, the lack of significant differences between the tested lines in their levels of malonylated ACC would not be surprising. To confirm the assumptions that D-Ala influences the D-Met stimulated ethylene production by inhibiting the ACC oxidase, the ACC malonyl transferase or both enzymes, further physiological experiments with D-Ala and structural analogs like Dcycloserine are required. However, the final answer to this question is awaited by the identification of the ACC malonyl transferase and the results of the enzyme's biochemical and physiological characterization.

Undoubtedly, AtDAT1 affects D-Met stimulated ethylene production and seems to have quite specific effects in this regard. However, as the working model in Figure 9 implies, the relationship between D-Met and ethylene may be more complex than just the competition for the $\mathrm{N}$-malonyl transferase (NMT). As mentioned above, D-Met may affect the levels of ACC and all its derivatives. It has been shown before that ACC itself acts as a signaling molecule and the same is also discussed for its derivatives (for reviews, see Van de Poel and Van Der Straeten, 2014; Vanderstraeten and Van Der Straeten, 2017; Nascimento et al., 2018). D-Met accumulation leads to an increase of ethylene concentrations, but possibly other compounds like ACC and its derivatives may also contribute to the observed physiological responses of dat1 affected plants. This would explain why the dat 1 mutants do not show the full spectrum of triple response after treatment in the presence of $\mathrm{D}$ Met. Detailed flux measurements of ACC and its derivatives after D-Met application as well as studies of dat1 alleles in the background of ethylene synthesis and receptor mutants may shed more light on this aspect.

In this regard also the intracellular localization of these biochemical processes is of interest. The localization of AtDAT1 implies that the transamination takes place in the chloroplast, whereas the ACC oxidation is postulated to happen either in the cytosol or the plasma membrane (Houben and Van De Poel, 2019). The separation of these processes raises the question how D-Met affects malonylation of ACC if it is also located in the cytosol. The most apparent hypothesis would be that chloroplasts have a certain capacity to take up D-Met. Flooding of the chloroplasts with this compound could therefore lead to inhibitory processes in the cytosol. This would be supported by the findings in this study that AtDAT1 is the major enzyme to degrade D-Met but needs further confirmation.

Another remaining question is the source of D-Met in nature, because it was not reported in plants until now. In contrast, it was demonstrated previously that D-Met is released by bacterial biofilms into the environment (Kolodkin-Gal et al., 2010; Vlamakis et al., 2013) and that different rhizosphere colonizing bacterial species are able to utilize D-Met as sole carbon and nitrogen source (Radkov et al., 2016). Biofilm formation on root surfaces as a bacterial pathogen protection strategy was reported before (Vlamakis et al. (2013). It is remarkable that D-Met is released by different bacterial species into their growth media to concentrations up to 300-500 $\mu \mathrm{M}$ (Lam et al., 2009), which would match the most effective D-Met concentrations in our study. Possibly, AtDAT1 is part of bacterial biofilm recognition and therefore may be involved in plant-bacterial interaction.

This possibility would also offer an explanation why AtDAT1 is dispensable in particular Arabidopsis accessions such as Ler and M7323S. An explanation for the dispensability of the D-Met catabolic function of AtDAT1 would be that in a habitat with only minor D-Met releasing bacteria in the rhizosphere, a recognition system for this compound would be also dispensable for the plant. However, this needs to be tested. The viability of Arabidopsis dat1 mutants and accessions without functional AtDAT1 also argues against the crucial function of this enzyme in folate biosynthesis. This was implied by the observation that the only known enzyme able to synthesize $\mathrm{p}$ amino benzoic acid (pABA), the substructure of folates, is AtDAT1 (Basset et al., 2004; Hanson and Gregory III, 2011). Consequently, the loss of this enzyme would lead to the inability to produce essential folate which would reduce the plant viability dramatically. Interestingly, $\mathrm{pABA}$ is also involved in the regulation of root gravitropism (Nziengui et al., 2018). This implies a modulatory role of AtDAT in differential root growth including gravitropism, which can be tested in future by the analysis of our dat1 mutants and accessions without or reduced AtDAT1 activity. Interestingly, DAT1 encoding genes seem to be found in all sequenced plant genomes (for a selection, see Figure S5), and ethylene production in other plant species than 
Arabidopsis is also induced by other D-AAs like D-Leu, D-Thr, D-Val, or D-Phe (Satoh and Esashi, 1980; Satoh and Esashi, 1982; Liu et al., 1983). In this regard, it would be interesting if also other D-AAs than D-Met cause growth defects and ethylene production in dat 1 mutants. Furthermore, it should be tested if the DAT1 enzymes from different species have altered substrate specificities and therefore contribute to the adaptation of plants to changing microbial environments.

\section{DATA AVAILABILITY STATEMENT}

All datasets generated for this study are included in the article/ Supplementary Material.

\section{AUTHOR CONTRIBUTIONS}

ÜK, JS, and $\mathrm{CH}$ designed the study. JS and $\mathrm{CH}$ conducted most of the experiments and contributed equally to the study. V-AL and $\mathrm{SH}$ conducted another part of the experiments. $\mathrm{CH}$ and MS analyzed the biochemical data and ÜK wrote the manuscript.

\section{REFERENCES}

Aldag, R., and Young, J. (1970). D-Amino Acids in Soils. I. Uptake and Metabolism by Seedling Maize and Ryegrass. Agron. J. 62, 184-189. doi: 10.2134/agronj1970.00021962006200020002x

Amelung, W., and Zhang, X. (2001). Determination of amino acid enantiomers in soils. Soil Biol. Biochem. 33, 553-562. doi: 10.1016/S0038-0717(00)00195-4

Amelung, W., Zhang, X., and Flach, K. (2006). Amino acids in grassland soils: climatic effects on concentrations and chirality. Geoderma 130, 207-217. doi: 10.1016/j.geoderma.2005.01.017

Balu, D. T., and Coyle, J. T. (2015). The NMDA receptor 'glycine modulatory site' in schizophrenia: D-serine, glycine, and beyond. Curr. Opin. Pharmacol. 20, 109-115. doi: 10.1016/j.coph.2014.12.004

Basset, G. J., Ravanel, S., Quinlivan, E. P., White, R., Giovannoni, J. J., Rébeillé, F., et al. (2004). Folate synthesis in plants: the last step of the p-aminobenzoate branch is catalyzed by a plastidial aminodeoxychorismate lyase. Plant J. 40, 453-461. doi: 10.1111/j.1365-313X.2004.02231.x

Benichou, M., Martinez-Reina, G., Romojaro, F., Pech, J. C., and Latché, A. (1995). Partial purification and properties of a $36-\mathrm{kDa} 1$-aminocyclopropane-1carboxylate N-malonyltransferase from mung bean. Physiol. Plant. 94, 629634. doi: 10.1111/j.1399-3054.1995.tb00977.x

Brückner, H., and Westhauser, T. (2003). Chromatographic determination of Land D-amino acids in plants. Amino Acids 24, 43-55. doi: 10.1007/s00726-0020322-8

Brückner, H. (2011). D-amino acids in chemistry, life sciences, and biotechnology (Zürich, Switzerland: Verlag Helvetica Chimica Acta).

Brodowski, S., Amelung, W., Lobe, I., and Du Preez, C. C. (2005). Losses and biogeochemical cycling of soil organic nitrogen with prolonged arable cropping in the South African Highveld-evidence from D-and L-amino acids. Biogeochemistry 71, 17-42. doi: 10.1007/s10533-005-5733-7

Brunhuber, N. M., Mort, J. L., Christoffersen, R. E., and Reich, N. O. (2000). Steadystate kinetic mechanism of recombinant avocado ACC oxidase: initial velocity and inhibitor studies. Biochemistry 39, 10730-10738. doi: 10.1021/bi0000162

Cava, F., Lam, H., De Pedro, M. A., and Waldor, M. K. (2011). Emerging knowledge of regulatory roles of D-amino acids in bacteria. Cell. Mol. Life Sci. 68, 817-831. doi: 10.1007/s00018-010-0571-8

\section{FUNDING}

JS was supported by the Deutscher Akademischer Austauschdienst (DAAD 91567028). We acknowledge support by Open Access Publishing Fund of University of Tübingen.

\section{ACKNOWLEDGMENTS}

We would like to acknowledge Prof. Klaus Harter (Center for Plant Molecular Biology, University of Tübingen, Germany) for the provision of research facilities and his invaluable help in the writing of this manuscript. Furthermore, we would like to thank Nina Glöckner and Friederike Wanke for their excellent support in confocal fluorescence microscopy and Georg Felix for his support in ethylene measurements.

\section{SUPPLEMENTARY MATERIAL}

The Supplementary Material for this article can be found online at: https://www.frontiersin.org/articles/10.3389/fpls.2019. 01609/full\#supplementary-material

Charng, Y.-Y., Chou, S.-J., Jiaang, W.-T., Chen, S.-T., and Yang, S. F. (2001). The catalytic mechanism of 1-aminocyclopropane-1-carboxylic acid oxidase. Arch. Biochem. Biophys 385, 179-185. doi: 10.1006/abbi.2000.2138

Chen, C., Maccready, J. S., Ducat, D. C., and Osteryoung, K. W. (2018). The molecular machinery of chloroplast division. Plant Physiol. 176, 138-151. doi: 10.1104/pp.17.01272

Chick, W., and Leung, P. (1997). lmmuno-purification and characterization of a $40 \mathrm{kDa}$ ACC N-malonyl transferase from mungbean seedling hypocotyl. Plant Physiol. 113, 119-126. doi: 10.1104/pp.113.1.119

Clough, S. J., and Bent, A. F. (1998). Floral dip: a simplified method for Agrobacterium-mediated transformation of Arabidopsis thaliana. Plant J. 16, 735-743. doi: 10.1046/j.1365-313x.1998.00343.x

Curtis, M. D., and Grossniklaus, U. (2003). A gateway cloning vector set for highthroughput functional analysis of genes in planta. Plant Physiol. 133, 462-469. doi: 10.1104/pp.103.027979

D'aniello, A. (2007). D-Aspartic acid: an endogenous amino acid with an important neuroendocrine role. Brain Res. Rev. 53, 215-234. doi: 10.1016/ j.brainresrev.2006.08.005

Dworkin, J. (2014). The medium is the message: interspecies and interkingdom signaling by peptidoglycan and related bacterial glycans. Annu. Rev. Microbiol. 68, 137-154. doi: 10.1146/annurev-micro-091213-112844

Edwards, K., Johnstone, C., and Thompson, C. (1991). A simple and rapid method for the preparation of plant genomic DNA for PCR analysis. Nucleic Acids Res. 19, 1349. doi: 10.1093/nar/19.6.1349

Erikson, O., Hertzberg, M., and Nasholm, T. (2004). A conditional marker gene allowing both positive and negative selection in plants. Nat. Biotechnol. 22, 455-458. doi: 10.1038/nbt946

Erikson, O., Hertzberg, M., and Näsholm, T. (2005). The dsdA gene from Escherichia coli provides a novel selectable marker for plant transformation. Plant Mol. Biol. 57, 425-433. doi: 10.1007/s11103-004-7902-9

Felix, G., Grosskopf, D. G., Regenass, M., Basse, C. W., and Boller, T. (1991). Elicitor-induced ethylene biosynthesis in tomato cells: characterization and use as a bioassay for elicitor action. Plant Physiol. 97, 19-25. doi: 10.1104/pp.97.1.19

Forde, B. G., and Roberts, M. R. (2014). Glutamate receptor-like channels in plants: a role as amino acid sensors in plant defence? F1000prime Rep. 6, 37 doi: 10.12703/P6-37 
Forsum, O., Svennerstam, H., Ganeteg, U., and Näsholm, T. (2008). Capacities and constraints of amino acid utilization in Arabidopsis. New Phytol. 179, 10581069. doi: 10.1111/j.1469-8137.2008.02546.x

Fuchs, S. A., Berger, R., Klomp, L. W., and De Koning, T. J. (2005). D-amino acids in the central nervous system in health and disease. Mol. Genet. Metab. 85, 168-180. doi: 10.1016/j.ymgme.2005.03.003

Fujii, N., Kaji, Y., and Fujii, N. (2011). d-Amino acids in aged proteins: analysis and biological relevance. J. Chromatogr. B 879, 3141-3147. doi: 10.1016/ j.jchromb.2011.05.051

Fujii, N. (2002). D-amino acids in living higher organisms. Origins Life Evol. Biosphere 32, 103-127. doi: 10.1023/A:1016031014871

Funakoshi, M., Sekine, M., Katane, M., Furuchi, T., Yohda, M., Yoshikawa, T., et al. (2008). Cloning and functional characterization of Arabidopsis thaliana d-amino acid aminotransferase-d-aspartate behavior during germination. FEBS J. 275, 1188-1200. doi: 10.1111/j.1742-4658.2008.06279.x

Gördes, D., Kolukisaoglu, U., and Thurow, K. (2011). Uptake and conversion of D-amino acids in Arabidopsis thaliana. Amino Acids 40, 553-563. doi: 10.1007/ s00726-010-0674-4

Gördes, D., Koch, G., Thurow, K., and Kolukisaoglu, U. (2013). Analyses of Arabidopsis ecotypes reveal metabolic diversity to convert D-amino acids. Springerplus 2, 559. doi: 10.1186/2193-1801-2-559

Gibson, E. J., Zhang, Z., Baldwin, J. E., and Schofield, C. J. (1998). Substrate analogues and inhibition of ACC oxidase: conversion of D-valine to isobutanal. Phytochemistry 48, 619-624. doi: 10.1016/S0031-9422(97)00965-5

Grefen, C., Donald, N., Hashimoto, K., Kudla, J., Schumacher, K., and Blatt, M. R. (2010). A ubiquitin-10 promoter-based vector set for fluorescent protein tagging facilitates temporal stability and native protein distribution in transient and stable expression studies. Plant J. 64, 355-365. doi: 10.1111/ j.1365-313X.2010.04322.x

Hamase, K., Morikawa, A., and Zaitsu, K. (2002). D-Amino acids in mammals and their diagnostic value. J. Chromatogr. B 781, 73-91. doi: 10.1016/S1570-0232 (02)00690-6

Hamase, K. (2007). Sensitive two-dimensional determination of small amounts of $\mathrm{D}$-amino acids in mammals and the study on their functions. Chem. Pharm. Bull. 55, 503-510. doi: 10.1248/cpb.55.503

Hanson, A. D., and Gregory, J. F. III. (2011). Folate biosynthesis, turnover, and transport in plants. Annu. Rev. Plant Biol. 62, 105-125. doi: 10.1146/annurevarplant-042110-103819

Hill, P. W., Quilliam, R. S., Deluca, T. H., Farrar, J., Farrell, M., Roberts, P., et al. (2011). Acquisition and assimilation of nitrogen as peptide-bound and Denantiomers of amino acids by wheat. PloS One 6, e19220. doi: 10.1371/ journal.pone.0019220

Hirano, T., Tanidokoro, K., Shimizu, Y., Kawarabayasi, Y., Ohshima, T., Sato, M., et al. (2016). Moss chloroplasts are surrounded by a peptidoglycan wall containing D-amino acids. Plant Cell 28, 1521-1532. doi: 10.1038/1841296b0

Hofstee, B. (1959). Non-inverted versus inverted plots in enzyme kinetics. Nature 184, 1296. doi: $10.1038 / 1841296$ b0

Houben, M., and Van De Poel, B. (2019). 1-Aminocyclopropane-1-carboxylic acid oxidase (ACO): the enzyme that makes the plant hormone ethylene. Front. Plant Sci. 10, 1-15. doi: 10.3389/fpls.2019.00695

Katane, M., and Homma, H. (2011). d-Aspartate-an important bioactive substance in mammals: a review from an analytical and biological point of view. J. Chromatogr. B 879, 3108-3121. doi: 10.1016/j.jchromb.2011. 03.062

Kionka, C., and Amrhein, N. (1984). The enzymatic malonylation of 1aminocyclopropane-1-carboxylic acid in homogenates of mung-bean hypocotyls. Planta 162, 226-235. doi: 10.1007/BF00397444

Kolodkin-Gal, I., Romero, D., Cao, S., Clardy, J., Kolter, R., and Losick, R. (2010). $\mathrm{D}$-amino acids trigger biofilm disassembly. Science 328, 627-629. doi: 10.1126/ science. 1188628

Konno, R., Brückner, H., D’Aniello, A., Fischer, G., Fujii, N., and Homma, H. (Eds.) (2007). d-Amino acids - a new frontier in d-Amino acids and protein research. Pract Meth Protocols (New York, NY, USA: Nova Science Publishers). 151-182.

Lam, H., Oh, D.-C., Cava, F., Takacs, C. N., Clardy, J., De Pedro, M. A., et al. (2009). D-amino acids govern stationary phase cell wall remodeling in bacteria. Science 325, 1552-1555. doi: 10.1126/science.1178123
Le Deunff, E., and Lecourt, J. (2016). Non-specificity of ethylene inhibitors: 'double-edged'tools to find out new targets involved in the root morphogenetic programme. Plant Biol. 18, 353-361. doi: 10.1111/plb.12405

Lempe, J., Balasubramanian, S., Sureshkumar, S., Singh, A., Schmid, M., and Weigel, D. (2005). Diversity of flowering responses in wild Arabidopsis thaliana strains. PloS Genet. 1, e6. doi: 10.1371/journal.pgen.0010006

Ling-Yuan, S., Yu, L., and Shang, F. Y. (1985). Relationship between 1aminocyclopropanecarboxylate malonyltransferase and D-amino acid malonyltransferase. Phytochemistry 24, 1141-1145. doi: 10.1016/S0031-9422 (00)81090-0

Liu, Y., Hoffman, N. E., and Yang, S. F. (1983). Relationship between the malonylation of 1-aminocyclopropane-1-carboxylic acid and D-amino acids in mung-bean hypocotyls. Planta 158, 437-441. doi: 10.1007/BF00397737

Liu, Y., Su, L.-Y., and Yang, S. F. (1985). Ethylene promotes the capability to malonylate 1aminocyclopropane-1-carboxylic acid and D-amino acids in preclimacteric tomato fruits. Plant Physiol. 77, 891-895. doi: 10.1104/pp.77.4.891

Magnani, G., Lomazzi, M., and Peracchi, A. (2013). Completing the folate biosynthesis pathway in Plasmodium falciparum: p-aminobenzoate is produced by a highly divergent promiscuous aminodeoxychorismate lyase. Biochem. J. 455, 149-155. doi: 10.1042/BJ20130896

Martínez-Rodríguez, S., Martínez-Gómez, A. I., Rodríguez-Vico, F., ClementeJiménez, J. M., and Las Heras-Vázquez, F. J. (2010). Natural occurrence and industrial applications of D-amino acids: an overview. Chem. Biodivers. 7 , 1531-1548. doi: 10.1002/cbdv.200900245

Michard, E., Lima, P. T., Borges, F., Silva, A. C., Portes, M. T., Carvalho, J. E., et al. (2011). Glutamate receptor-like genes form $\mathrm{Ca} 2+$ channels in pollen tubes and are regulated by pistil D-serine. Science 332, 434-437. doi: 10.1126/ science.1201101

Monselise, E. B., Levkovitz, A., and Kost, D. (2015). Ultraviolet radiation induces stress in etiolated Landoltia punctata, as evidenced by the presence of alanine, a universal stress signal: a (1)(5)N NMR study. Plant Biol. (Stuttg) 17 Suppl 1, 101-107. doi: 10.1111/plb.12198

Naranjo-Ortíz, M. A., Brock, M., Brunke, S., Hube, B., Marcet-Houben, M., and Gabaldón, T. (2016). Widespread inter-and intra-domain horizontal gene transfer of d-amino acid metabolism enzymes in eukaryotes. Front. Microbiol. 7, 2001. doi: 10.3389/fmicb.2016.02001

Nascimento, F. X., Rossi, M. J., and Glick, B. R. (2018). Ethylene and 1Aminocyclopropane-1-carboxylate (ACC) in plant-bacterial interactions. Front. Plant Sci. 9, 114. doi: 10.3389/fpls.2018.00114

Nelson, B. K., Cai, X., and Nebenführ, A. (2007). A multicolored set of in vivo organelle markers for co-localization studies in Arabidopsis and other plants. Plant J. 51, 1126-1136. doi: 10.1111/j.1365-313X.2007.03212.x

Nziengui, H., Lasok, H., Kochersperger, P., Ruperti, B., Rébeillé, F., Palme, K., et al. (2018). Root gravitropism is regulated by a crosstalk between paraaminobenzoic acid, ethylene, and auxin. Plant Physiol. 178, 1370-1389. doi: 10.1104/pp.18.00126

Ollivaux, C., Soyez, D., and Toullec, J. Y. (2014). Biogenesis of D-amino acid containing peptides/proteins: where, when and how? J. Pept. Sci. 20, 595-612. doi: $10.1002 /$ psc.2637

Radkov, A. D., Mcneill, K., Uda, K., and Moe, L. A. (2016). D-Amino acid catabolism is common among soil-dwelling bacteria. Microbes Environ. 31, 165-168. doi: 10.1264/jsme2.ME15126

Satoh, S., and Esashi, Y. (1980). D-Amino-acid-stimulated ethylene production in seed tissues. Planta 149, 64-68. doi: 10.1007/BF00386229

Satoh, S., and Esashi, Y. (1982). Effects of $\alpha$-aminoisobutyric acid and D-and Lamino acids on ethylene production and content of 1-aminocyclopropane-1carboxylic acid in cotyledonary segments of cocklebur seeds. Physiol. Plant. 54, 147-152. doi: 10.1111/j.1399-3054.1982.tb06318.x

Strauch, R. C., Svedin, E., Dilkes, B., Chapple, C., and Li, X. (2015).Discovery of a novel amino acid racemase through exploration of natural variation in Arabidopsis thaliana. Proc. Natl. Acad. Sci. 112, 11726-11731. doi: 10.1073/ pnas. 1503272112

Thrower, J., Mirica, L. M., Mccusker, K. P., and Klinman, J. P. (2006). Mechanistic investigations of 1-aminocyclopropane 1-carboxylic acid oxidase with alternate cyclic and acyclic substrates. Biochemistry 45, 13108-13117. doi: 10.1021/ bi061097q 
Valdovinos, J. G., and Muir, R. M. (1965). Effects of D and L amino acids on foliar abscission. Plant Physiol. 40, 335. doi: 10.1104/pp.40.2.335

Van De Poel, B., and Van Der Straeten, D. (2014). 1-aminocyclopropane-1carboxylic acid (ACC) in plants: more than just the precursor of ethylene!. Front. Plant Sci. 5, 1-15. doi: 10.3389/fpls.2014.00640

Vanderstraeten, L., and Van Der Straeten, D. (2017). Accumulation and transport of 1-aminocyclopropane-1-carboxylic acid (ACC) in plants: current status, considerations for future research and agronomic applications. Front. Plant Sci. 8, 38. doi: 10.3389/fpls.2017.00038

Vlamakis, H., Chai, Y., Beauregard, P., Losick, R., and Kolter, R. (2013). Sticking together: building a biofilm the Bacillus subtilis way. Nat. Rev. Microbiol. 11, 157. doi: $10.1038 /$ nrmicro2960

Vranova, V., Zahradnickova, H., Janous, D., Skene, K. R., Matharu, A. S., Rejsek, K., et al. (2012). The significance of D-amino acids in soil, fate and utilization by microbes and plants: review and identification of knowledge gaps. Plant Soil 354, 21-39. doi: 10.1007/s11104-011-1059-5

Winter, D., Vinegar, B., Nahal, H., Ammar, R., Wilson, G. V., and Provart, N. J. (2007). An "Electronic Fluorescent Pictograph" browser for exploring and analyzing largescale biological data sets. PloS One 2, e718. doi: 10.1371/journal.pone.0000718
Wu, Y. M., Silverstone, A. L., Liu, Y., and Yang, S. F. (1995). Partialpurification and characterization of tryptophan N-Malonyltransferase from tomato leaves. Phytochemistry 40, 699-704. doi: 10.1016/0031-9422(95) 00320-7

Yang, S. F., and Hoffman, N. E. (1984). Ethylene biosynthesis and its regulation in higher plants. Annu. Rev. Plant Physiol. 35, 155-189. doi: 10.1146/annurev. pp.35.060184.001103

Conflict of Interest: The authors declare that the research was conducted in the absence of any commercial or financial relationships that could be construed as a potential conflict of interest.

Copyright (c) 2019 Suarez, Hener, Lehnhardt, Hummel, Stahl and Kolukisaoglu. This is an open-access article distributed under the terms of the Creative Commons Attribution License (CC BY). The use, distribution or reproduction in other forums is permitted, provided the original author(s) and the copyright owner(s) are credited and that the original publication in this journal is cited, in accordance with accepted academic practice. No use, distribution or reproduction is permitted which does not comply with these terms. 